\title{
NIVEL DE SATISFACCIÓN EN LA FORMACIÓN DE CALIDAD A NIVEL UNIVERSITARIO
}

\author{
Nelly Dioses Lescano* \\ https://orcid.org/0000-0001-7889-1024 \\ Humberto Iván Morales Huamán* \\ https://orcid.org/0000-0002-8720-4959 \\ Doris Margot Díaz de Angulo* \\ https://orcid.org/0000-0001-9737-1593 \\ Antenor Vasquez Muñoz* \\ https://orcid.org/0000-0002-1554-1120
}

RECIBIDO: Enero 2021 / ACEPTADO: Febrero 2021 / PUBLICADO: Mayo 2021

Como citar: Dioses Lescano, Nelly; Morales Huamán, Humberto; Díaz de Angulo, Doris; Vasquez Muñoz, Antenor (2021). Nivel de satisfacción en la formación de calidad a nivel universitario. Telos: revista de Estudios Interdisciplinarios en Ciencias Sociales, 23 (2), Venezuela. (Pp.247-266).

DOI: www.doi.org/10.36390/telos232.04

\section{RESUMEN}

La presente investigación tiene como objetivo analizar cómo influye el nivel de satisfacción del estudiante en relación con la formación de calidad profesional que recibe a nivel universitario. Dado que resulta importante para la superación personal de cada estudiante lograr los conocimientos y habilidades que permitan un desarrollo de calidad en el área profesional en la cual van a desempeñarse a futuro y demostrar las capacidades aprendidas. Los autores que fundamentan teóricamente el presente estudio son: Teo (2019), Belasha, et al. (2015), Benavides (2018), entre otros. Metodologícamente se utilizó como instrumento de recolección de datos la técnica de la encuesta validada y diseñada con la escala de Likert, la cual fue aplicada a una muestra de 7180 estudiantes de diferentes facultades en forma aleatoria simple, siendo la población total conformada por todos los estudiantes de la universidad Señor de Sipán en el año 2019. Donde se pudo obtener como resultados que más del $83.2 \%$ de los estudiantes universitarios evidencian satisfacción en la calidad en la formación profesional

\footnotetext{
* Doctora en Ciencias de la Educación, docente de la Universidad Señor de Sipán, Chiclayo-Perú, Correo Electrónico: nellydioses21@gmail.com

* Doctor en Administración de la Educación, docente de la Universidad Señor de Sipán, Chiclayo - Perú, Correo Electrónico: ivanmoraleshu@gmail.com

* Doctora, dentro del Programa de Investigación e Innovación Educativa de la Universidad de Málaga, docente de la Universidad Señor de Sipán, Chiclayo- Perú, Correo Electrónico: diazcastillodoris@gmail.com

* Doctor en Ciencias de la Educación, docente de la Universidad Señor de Sipán, Chiaclayo-Perú, Correo Electrónico: antenorvasquez_14@hotmail.com
} 
recibida. Concluyendo, que a mayor nivel de satisfacción que perciben los estudiantes universitarios en los diferentes servicios que brinda la universidad, más se puede influenciar positivamente sobre la calidad de la formación profesional.

Palabras clave: Nivel de satisfacción, formación de calidad, calidad de servicio, superación personal, estudiante universitario.

\title{
Level of satisfaction on quality training at the university level
}

\begin{abstract}
The present research aims to analyze how the level of student satisfaction influences in relation to the professional quality training received at the university level. Since it is important for the personal improvement of each student to achieve the knowledge and skills that allow quality development in the professional area in which they will perform in the future and demonstrate the skills learned. The authors who theoretically support this study are: Teo (2019), Belasha, Popov, et al. (2015), Benavides (2018), among others. Methodologically, the validated survey technique designed with the Likert scale was used as a data collection instrument, which was applied to a sample of 7180 students from different faculties in a simple random way, the total population being made up of all students. from Señor de Sipán University in 2019. Where it was possible to obtain as results that more than $83.2 \%$ of university students show satisfaction in the quality of the professional training received. Concluding that the higher the level of satisfaction perceived by university students in the different services offered by the university, the more it can positively influence the quality of professional training.
\end{abstract}

Keywords: Level of satisfaction, quality training, quality of service, self-improvement, university student.

\section{Introducción}

La globalización está induciendo cambios a nivel mundial en todos los niveles sociales, producto del desarrollo tecnológico, el Perú está viviendo un desarrollo científico que nunca antes ha experimentado, el cual requiere nuevas estrategias para poder enfrentar con éxitos dichos avances científicos. Por ello, se tiene que partir de una educación de calidad, donde al estudiante no solo se le de el servicio óptimo; sobre todo que lo valore para lograr una formación de calidad en su proceso formativo.

Es muy importante que el estudiante universitario este satisfecho con la calidad de educación que le brinda su institución, demostrando con esto cuanto valora la universidad la formación profesional de sus estudiantes, reflejado mediante políticas, estratégicas de servicios a nivel académico y administrativo que les brinda a cada uno de ellos. Se necesita una universidad que además de infraestructura, laboratorios, biblioteca e instalaciones de calidad, ofrezca un buen clima institucional, donde se evidencie que la razón de ser es el estudiante y no los intereses personales, ni políticos que daña la imagen y la calidad educativa.

Sin embargo, Alvarado y Moreno (2017), el sistema de gestión se hace más complejo e ineficiente a medida que las universidades aumentan de tamaño, debido al incremento del número de alumnos y profesores como respuesta a una demanda continuamente insatisfecha, 
multiplican sus funciones, crece el personal administrativo, de apoyo y por lo tanto aumentan sus gastos, dejando de lado el fortalecimiento de la investigación y su vinculación con los actores sociales involucrados en el sistema nacional de ciencia y tecnología.

Por lo tanto, en América Latina muchas universidades han tenido que reorientar sus políticas de gestión, direccionadas a mejorar la planificación, organización, ejecución y control, por parte de las instituciones del Estado que deben exigir educación de calidad en el sector educativo. A nivel universitario en el Perú, actualmente se ofrecen varias modalidades de estudios, sin embargo, muchas de ellas no responden completamente a las expectativas de la sociedad y además no están acordes a las exigencias laborales de los perfiles de los egresados.

En este sentido, las universidades deben preocuparse por la calidad educativa que imparten, realizando una óptima planificación, organización, ejecución, control y evaluación permanente, respondiendo mejor a las expectativas de los estudiantes. Lo cual permite mejorar la calidad educativa, formando profesionales proactivos, innovadores, competentes y de alta competitividad, capaces de competir en un mercado laboral globalizado. Sin embargo, para conocer el nivel de calidad de servicio que las universidades imparten se debe medir mediante el grado de satisfacción de los estudiantes que presentan con la institución educativa donde están estudiando o ya estudiaron una carrera profesional.

Debido a las pocas investigaciones que existen sobre el tema que se aborda en el presente estudio, se busca por lo tanto, analizar cómo influye el nivel de satisfacción del estudiante en relación con la formación de calidad profesional que recibe a nivel universitario, a fin de visualizar donde están las principales fallas del sistema educativo universitario y ofrecer lineamientos que permitan mantener la calidad de servicio que se le brinda a sus estudiantes, contribuyendo con una mejor formación profesional en función a las competencias que deben desarrollar para cumplir con las exigencias del campo laboral tanto a nivel público como privado, optimizando de forma continua los servicios que ofrecen las universidades en el Perú.

\section{Educación universitaria de calidad.}

Hoy en día, la calidad de la educación superior es uno de los problemas más importantes del desarrollo social, del progreso científico y tecnológico, según lo refiere Trujillo (2014), la importancia de mejorar la calidad de la educación superior se atribuye principalmente a los requisitos de una economía innovadora basada en el conocimiento y las necesidades del individuo y la sociedad para mejorar la competitividad y la calidad de vida. Al respecto agregan Şen, et al. (2012), que para que la universidad desarrolle una educación de alta calidad es necesario la democratización de las prácticas de gestión universitaria.

Adicionalmente, Rivero (2013), afirma que es común considerar a la educación desvinculada de los fenómenos políticos, económicos y sociales más amplios. Por su parte, agregan Brunner y Ganga (2018), la educación superior se halla propulsada por cuatro vectores de fuerza que se combinan de diferentes maneras y en proporciones variables: el Estado, los mercados, las propias corporaciones y la sociedad civil.

De manera que el desafío para la política pública y para las normas que deben regir a la educación superior de un país es cómo esas fuerzas se coordinan, para crear un entorno favorable dentro de las instituciones de educación superior. Conforme a lo planteado se ha podido evidenciar que en la actualidad en el Perú existen varias universidades, que no ofrecen 
una educación de calidad, por lo que, se debe invertir más y optimizar los servicios a nivel educativo y administrativo; como lo destacan Belasha, et al. (2015), el componente esencial de una evaluación y garantía de calidad de un programa de educación es el monitoreo de la satisfacción de los interesados con los resultados educativos.

El autor Vilcea (2014), afirma que la calidad en la educación superior es un concepto complejo y hasta ahora es difícil establecer un enfoque generalmente aceptado. La educación universitaria de calidad en el Perú debe estar plasmada en un proyecto que pertenezca al plan institucional, donde estratégicamente se cumpla gradualmente con lo planificado para alcanzar las metas y así brindar a los estudiantes educación acorde con sus objetivos personales, tomando en cuenta el fenómeno tecnológico que vive la humanidad. Para ello, la universidad tiene que tener infraestructura, plana jerárquica, docentes, administrativos, políticas y alumnos de calidad.

Cuando la universidad quiere evaluar el nivel de satisfacción de los estudiantes frente a los servicios que ella ofrece, es muy subjetivo depende de muchas variables como el estado anímico, motivación, madurez, percepción, responsabilidad, comparación con otras instituciones. Bulut, Serin y Özbaş (2010), analizan en qué medida el nivel de satisfacción con la vida de los estudiantes universitarios puede influenciar con la percepción sobre calidad del nivel de educación universitaria. Por su parte, Motefakker (2016), recomienda que las autoridades universitarias deben tomar medidas pertinentes para aumentar la satisfacción de los estudiantes, mejorando el nivel de servicios educativos de calidad. Shaikh, et al, (2019), afirman que los docentes necesitan ampliar sus estrategias de instrucción, a fin de construir un ambiente de aprendizaje de calidad.

No obstante, la universidad como institución educativa debe implementar los estándares de ISO 9001, homogeneizando los criterios que sirvan de base para medir el grado de satisfacción de los estudiantes y la influencia en su formación académica. Según Sutherlanda y Warwicka (2019), comprender los vínculos entre la satisfacción del estudiante y la calidad de la enseñanza a nivel universitario es una preocupación en muchas instituciones educativas. Foon, et al (2020), afirman que el docente, el contenido, la evaluación y el horario juegan un papel importante en la satisfacción del estudiante, mientras que la estructura del curso, especialización, duración, interacción, carga de trabajo y dificultad percibida no juegan roles significativos.

Conforme a lo planteado, es importante destacar que las instituciones educativas deben responder a las expectativas de los estudiantes, porque si ellos no perciben satisfacción en la calidad educativa que reciben, pondrá en riesgo la calidad de su formación profesional, formando profesionales no competentes al mercado laboral, generando una insatisfacción en el egresado, sociedad y empleadores.

\section{Gestión de calidad a nivel universitario.}

En el Perú, las universidades están adecuándose a los procesos de gestión basada en estándares, para mejorar la calidad educativa en beneficio de los estudiantes y la sociedad. Presentándose como uno de los mayores desafíos en la gestión universitaria. En otras palabras, la gestión de calidad a nivel universitario, permite sistematizar políticas, objetivos, metas con el propósito de mejorar el nivel de calidad de formación profesional. 
Los criterios e indicadores de calidad son la piedra angular de los procesos de acreditación, tal como lo señala Cruz (2009), citado por Sánchez Quintero (2014), sobre ellos se construye todo un sistema, no solo el de garantía de la calidad, sino también el de educación superior. Un sistema de criterios e indicadores inadecuados traerá consigo un sistema de educación superior de baja calidad y por tanto inadecuado para solventar las necesidades sociales.

En este sentido, ISO 9001 es una norma reconocida internacionalmente que según Betlloch, et al, (2019), proporciona los recursos necesarios para ayudar a una organización a mejorar su rendimiento, basándose en el principio de planificar-hacer-controlar-actuar, con el fin de obtener una mejora continua. Las universidades están obligadas a responder a las expectativas de la sociedad, por ello, deben mejorar los procesos de gestión de calidad, frente a una sociedad del conocimiento que se encuentra en constante cambios sustanciales, evidenciándose dichas mejoras en la formación de calidad que se le brinda a los estudiantes universitarios y que los conocimientos impartidos se apliquen a solucionar problemas sociales.

Para la Real Academia Española (RAE, 2010), la palabra calidad es conceptualizada como la "prioridad o conjunto de prioridades inherentes a algo, que permite juzgar su valor" o como "superioridad o excelencia". Por ello, hablar de calidad educativa en la actualidad ya no es ajeno a las universidades, se ha convertido en un desafío permanente a resolver. Asimismo, cuando se habla de calidad Alvarado e Izaguirre (2015), señalan que contempla exceder las expectativas de los clientes, propiciar el desarrollo de sistemas de monitoreo a las diversas variantes del mercado, el cual, contiene un alto nivel de volatilidad y subjetividad.

Por lo cual, Barbón (2018), afirma que la integración de la gestión del conocimiento, la ciencia, la tecnología y la innovación como brújula de la gestión educativa estratégica en el ámbito universitario ha dado al traste con los antiguos límites que abrían brechas entre la investigación, el posgrado y la extensión universitaria. En este sentido, educación para todos, durante toda la vida, es el paradigma educativo que la UNESCO (1998),, ha establecido a fin de identificar el objetivo supremo al cual debe aspirar la sociedad moderna. Este recoge un anhelo, difícil de hacer realidad, al ignorar el rol de la gestión educativa estratégica en la integración de la gestión del conocimiento, la ciencia, la tecnología y la innovación en la educación.

Según García Jimenez (2016), la educación superior se ha convertido en un factor clave del progreso económico, cultural y social de cualquier país. Así, una de las funciones que se espera de las universidades es que promuevan y/o mantengan el desarrollo social, económico y cultural de las comunidades en las que están situadas a través de sus propuestas formativas, el impulso de la investigación aplicada y la transferencia de conocimiento.

A lo cual, señalan Gafas Gonzalez, et al., (2018), la preparación permanente e integral del docente universitario constituye un eje fundamental para la formación del estudiante y la transformación de la sociedad. Se sustenta en adquisición por estos de habilidades del lenguaje, la comunicación, el pensamiento crítico, ético y del conocimiento de un idioma extranjero y de las TIC, lo que les permitirá integrar las funciones sustantivas de la universidad, guiadas estas por la investigación.

A modo de reflexión, se puede decir, que la gestión de la calidad universitaria, es un proceso metodológico, que debe ser contextualizado al entorno, tiempo y exigencias de la sociedad, basado en principios de excelencia, calidad y pertinencia, buscando su subsitencia 
futura, en la cual un elemento importante para alcanzarla es ser competitivo mediante la calidad reconocida a partir de su acreditación, en otras palabras, es un proceso de mejoramiento continuo de la calidad universitaria, medido a través de la trascendencia y del impacto social de los resultados alcanzados.

\section{Retos para una educación universitaria de calidad en el siglo XXI.}

El Perú enfrenta en la actualidad muchos desafíos a nivel social, ambiental, económico, político, cultural, educativo entre otros. Frente a ello, el Estado tiene que responder con políticas claras, acciones creíbles, con equidad y trasparencia, garantizando el desarrollo integral para todos. En este sentido, Teo (2019), señala que en las últimas dos décadas, ha habido un llamado a los docentes de todo el mundo para preparar a los estudiantes para el siglo XXI ayudándolos a navegar por un mundo globalizado y un paisaje interconectado. A lo cual, agregan Adie, et al (2019), en la actualidad existe una mayor expectativa en las instituciones de educación superior; por tal razón, el sector educativo universitario viene realizando una serie de cambios, concepciones y paradigmas asumiendo nuevos retos.

Al respecto, Hernández, et al., (2017), exponen que una de las limitaciones hacia la educación de calidad son los desafíos socioeconómicos, particularmente sobre la desigualdad y la pobreza, porque compartimos la idea de que son obstáculos al crecimiento que impiden arribar a mejores niveles de vida, incluido el acceso a la educación en sus distintos niveles, Por lo cual, las universidades juegan un papel sustancial para superar estos grandes problemas.

Durante los últimos años, el mundo universitario está cambiando. Según García (2012), citado por Navea y Suarez (2017), las instituciones educativas son llamadas a formar parte de un nuevo modelo, en el que los estudiantes universitarios se convierten en futuros profesionales capaces de ser aprendices con una óptima capacidad de aprender a aprender durante toda la vida en un entorno globalizado.

El autor Osborne (2013), señala que la educación universitaria podría abordar los problemas contemporáneas de la sociedad, sin embargo, una deficiente calidad educativa dificulta tal propósito. Por ello, sin una educación de calidad en el presente siglo competitivo y global va ser imposible desterrar los problemas sociales que enfrenta la sociedad peruana. Sin educación de calidad la corrupción invade y la democracia puede ser vista como una utopía difícil de conseguir, la igualdad de oportunidades pueden alcanzarlo solo unos pocos, una élite que se cree con derechos.

Mediante la educación universitaria de calidad para todos se puede corregir problemas sociales que se viene heredando por varios siglos, realidad que muchos gobiernos de turno no lo atendido como prioridad y política de Estado. Evidenciándose en la actualidad como una prioridad para los gobernantes y un reclamo permanente de la sociedad peruana. Las universidades contribuyen al crecimiento del país de una manera significativa, en ella se forman los nuevos profesionales que el mercado laboral requiere.

Al respecto, Gaete Quezada (2015), la educación superior ha sido por décadas un ámbito social donde las mujeres paulatinamente han logrado importantes espacios de inserción y participación, especialmente en lo relativo al ingreso a los estudios universitarios que han evolucionado desde un acceso absolutamente orientado a los hombres de nivel socioeconómico alto. En función a lo planteado por el autor antes mencionado, se puede decir que los retos que tienen las universidades en el Perú son trascendentales, tienen que estar 
constantemente evaluando como responder a la sociedad y no funcionar fuera de ella, tiene que ser universidades que solucionen problemas sociales y no ser parte del problema.

A su vez, plantean Bruns, Harbaug y Schneider (2019), en primer lugar, los retos de cambio a nivel universitario generalmente afectan los intereses institucionales de sindicatos de docentes bien organizados y políticamente poderosos. En segundo lugar, la implementación es opaca, ya que el impacto depende del cambio en el aula que es difícil de monitorear para los reformadores. Y, tercero, los beneficios son a largo plazo, generalmente mucho más allá de la tenencia política de los defensores de la reforma. Se requieren universidades que cambien los destinos de la patria, siendo esto un desafío a lograr en el presente siglo.

Asimismo, durante los últimos años las universidades en el Perú están enfrentando retos que no fueron planificados por muchas de ellas, atravesando el fenómeno de la intercionalización realidad que ya no pueden evadir, al contrario las universidades tienen el reto de fortalecerse mejorando permanente la calidad educativa, por medio de la adecuación de nuevos planes curriculares, pensando siempre en la razón de ser que es el estudiante, sujeto de cambio.

Por lo tanto, las universidades peruanas del sector público y privado tienen el reto de adecuar sus políticas educativas y trabajar en cooperación permanente, para responder mejor al fenómeno de la globalización, teniendo todas las universidades un objetivo en común: mejorar el nivel de la calidad educativa pensando en el futuro profesional y en el país. Donde no solamente se quede en acuerdos y convenios sino que se evidencia en la realidad porque los beneficiados serían todos los peruanos.

\section{Importancia de las reformas universitarias en el Perú.}

En el Perú contemporáneo, de acuerdo a lo planteado por Mejía (2018), el mercantilismo marca la pauta general del patrón universitario como parte del proceso de globalización neoliberal que cubre la sociedad. No obstante, la educación superior es resultado de un proceso de transacción de varias trayectorias de universidad que la definen y señalan un momento crucial de disputa sobre la organización de la producción cognoscitiva.

Según, Buchbinder (2018), la aspiración siempre explícita de los reformistas, es construir una Universidad mejor, con un compromiso más intenso con la vida cultural y científica de las sociedades. Se trataba de una Universidad menos comprometida con la formación individual de los profesionales, con el usufructo personal de los beneficios que brindan los títulos y, en cambio, mucho más intimamente articulada con la tarea de construir una sociedad más igualitaria y más justa.

En los últimos 20 años en el Perú se permitió llevar a cabo la tarea antes mencionada para satisfacer la demanda de una mejor calidad educativa a nivel de educación básica regular y universitaria, a diversas empresas nacionales y extranjeras, porque el Estado es incapaz de solucionar esta necesidad. El crecimiento de ofertas educativas no estuvo acompañado siempre de la calidad, generando incongruencia frente a la expectativa de la sociedad peruna, por ello el Estado como ente regulador, tuvo que aprobar desde el congreso una nueva ley unversitaria No. 30220 para regular y mejorar los estándares de calidad educativa universitaria.

Dicho proceso se está ejecutando a nivel nacional en todas las universidades nacionales y particulares, exigiendo a las universidades un mejor servicio educativo; se conoce con el nombre de licenciamiento: el cual es un mecanismo obligatorio para todas las universidades, 
donde se evalua las diferentes condiciones básicas de calidad referidas al equipamiento, infraestructura, áreas culturales y bolsa de trabajo, un plan de estudios adecuado a la Ley Universitaria, así como con un vicerrectorado de investigación, para lograr tales caracteristicas este organismo denominado SUNEDU primero sensibilizó y capacitó a las universidades que lo solicitaban, luego hizo llegar las condiciones básicas, para ser implemetadas.

En la actualidad se ha avanzado mucho en relación a las universidades que han sido licenciadas por un periodo de tiempo y otras han sido denegadas el licenciamiento, por lo que tienen un plazo de dos años para dejar de ofrecer tal servicio educativo. Especificamente, según Benavides Abanto (2018), titular de la Sunedu se contabilizan 36 universidades las que han aprobado la supervisión y obtenido su licenciamiento.

En general, las reformas univeritarias han sido resultado de un proceso de transición durante varios años, definiendo las mismas como calificadas y buscando ser acreditadas y reconocidas internacionalmente por sus planes de estudio, infraestructura de la institución, plantilla docente, acciones de investigación, actividades complementarias y de responsabilidad social que busquen responder a las exigencias sociales, entre otros elementos que son requeridos para ofrecer un mejor servicio educativo de calidad.

\section{Metodología.}

La investigación es descriptiva, debido a que busca describir la satisfacción de los estudiantes en la formación de calidad que reciben a nivel universitario, con el propósito de ser analizada, para que las universidades mejoren la calidad y percepción por parte de los estudiantes de los servicios educativos que éstos reciben. Por lo cual, la investigación también se considera analítica, según Argimón Pallás y Jiménez Villa (2014), contrasta las hipótesis de trabajo definidas previamente, tiene una orientación explicativa, busca comprender el porqué de los fenómenos, o bien predictiva, en el sentido de intentar hacer predicciones fiables y brindar la posibilidad de control sobre determinados problemas, en este caso es el nivel de formación de calidad.

Por otra parte, la investigación tiene un diseño no experimental de campo, conforme a los planteamientos de Arias Odón (2016), ya que no se manipularán las variables y se consultará directamente a las unidades de observación. Igualmente, se pretende recabar la información en un solo momento, correspondiendo esto último a un estudio transaccional. La investigación es de carácter cuantitativa debido a que recoge información mediante las encuestas realizado a los estudiantes de la universidad Señor de Sipán para demostrar la hipótesis que se plantea en la investigación. Dicha hipótesis se presenta a continuación:

Hipótesis: Si se evaluaría el nivel de satisfacción en el estudiante; entonces, se mejoraría la calidad de formación profesional en la universidad Señor de Sipán formando profesionales más competetivos.

Problema: ¿Cuál es la relación que existe entre el nivel de satisfacción del servicio educativo para el estudiante con la calidad de formación profesional en la universidad Señor de Sipán?

La población que fue considerada para el desarrollo de la presente investigación, corresponde a 16200 estudiantes de la universidad Señor de Sipán, de ambos sexos, de las cinco Facultades y de diferentes ciclos académicos. Dado que la población es amplia, se requirió de una muestra conformada por 7180 estudiantes, los cuales fueron entrevistados de forma aleatoria simple al azar, durante un mes, utilizando para ello el apoyo de 10 
encuestadores a razón de 30 encuestados diarios por cada entrevistador, logrando así que todos los entrevistados respondieran la totalidad de las interrogantes pautadas en el instrumento de recolección de datos. La técnica empleada fue la encuesta conformada por un total de 36 preguntas. El instrumento utilizado fue cuestionario en la escala de Likert, correspondiente a 1 el nivel más bajo y 5 el más alto, tal como se muestra en la tabla 1.

Tabla No. 1. Escala de medición de las opciones de respuesta

\begin{tabular}{|c|c|c|c|c|}
\hline \multicolumn{5}{|c|}{ Valoraciones } \\
\hline 1 & 2 & 3 & 4 & 5 \\
Malo & Regular & Bueno & Muy bueno & excelente \\
\hline
\end{tabular}

Fuente: Elaboración propia

\section{Resultados y discusión}

A continuación se presentan los resultados obtenidos luego de aplicado el instrumento de recolección de datos en donde se puede evidenciar la influencia entre el nivel de satisfacción del estudiante en relación con la formación de calidad profesional que recibe a nivel universitario. Donde, se pudieron analizar dimensiones como las condiciones didácticas, institucionales, de calidad universitaria, de asistencia a clases, de interrelación estudiantedocente y de aptitudes que son aspectos fundamentales para medir la variable nivel de satisfacción y por lo tanto, analizar como es la calidad en la formación universitaria de Perú.

\section{Gráfico No. 1: Condiciones diadacticas}

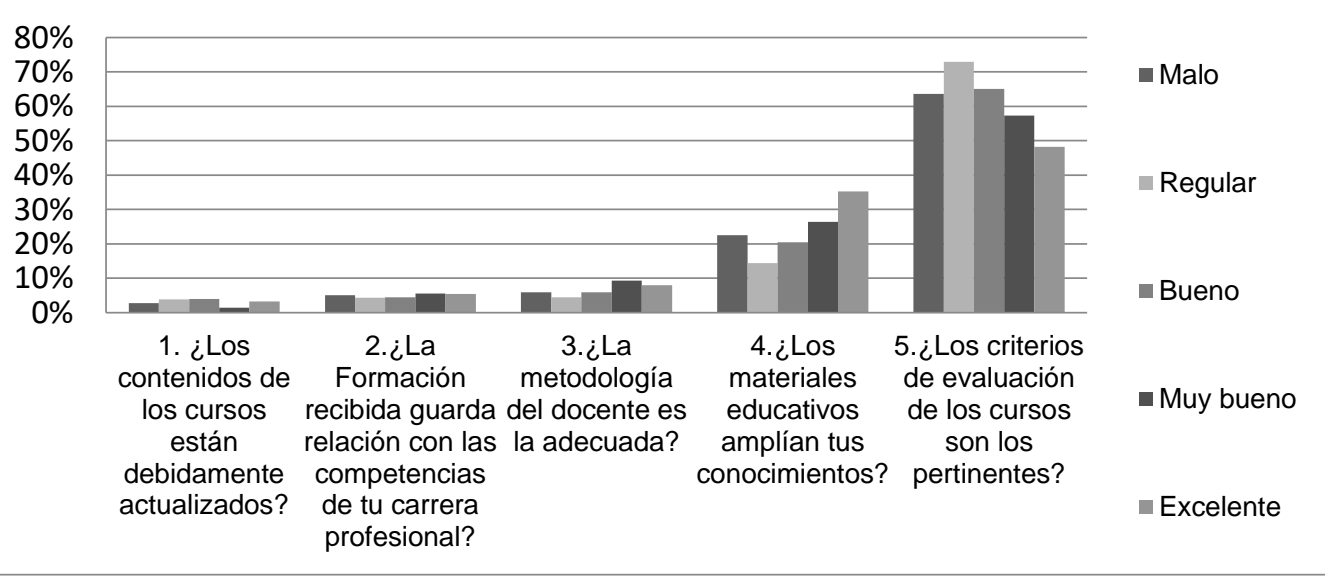

Fuente: Elaboración propia.

En el gráfico No. 1 se muestran los resultados obtenidos en relación a la dimensión condiciones didácticas que evidencian los estudiantes de la Universidad Señor de Sipán (USS), donde se puede ver como el $64 \%$ de los entrevistados notan que los contenidos de los cursos están debidamente actualizados, igualmente, el $73 \%$ de los estudiantes indican que la 
formación recibida guarda relación con las competencias de su carrera profesional, seguidamente, el $65 \%$ señala que la metodología del docente es la adecuada, al igual que el $57 \%$ de los estudiantes quienes indicaron que los materiales educativos amplían sus conocimientos y con respecto a si los criterios de evaluación de los cursos son los pertinentes, solo el $48 \%$ de los entrevistados manifestaron que son excelentes.

Los resultados mostrados en el gráfico No.1 demuestran que las condiciones didácticas actuales en las universidades de Perú, son excelentes, que existe calidad de formación en dichas instituciones, coincidiendo con lo planteado por Brewa y Saunders (2020), sobre el desafío que tienen los docentes a repensar las estrategias pedagógicas, en un mundo globalizado como el actual, para cumplir con las exigencias de la sociedad, asimismo Benavides (2018), habla de profesores calificados y programas actualizados para el desarrollo de competencias acordes a los requerimientos laborales y exigencias del estudiantado en materia de formación académica.

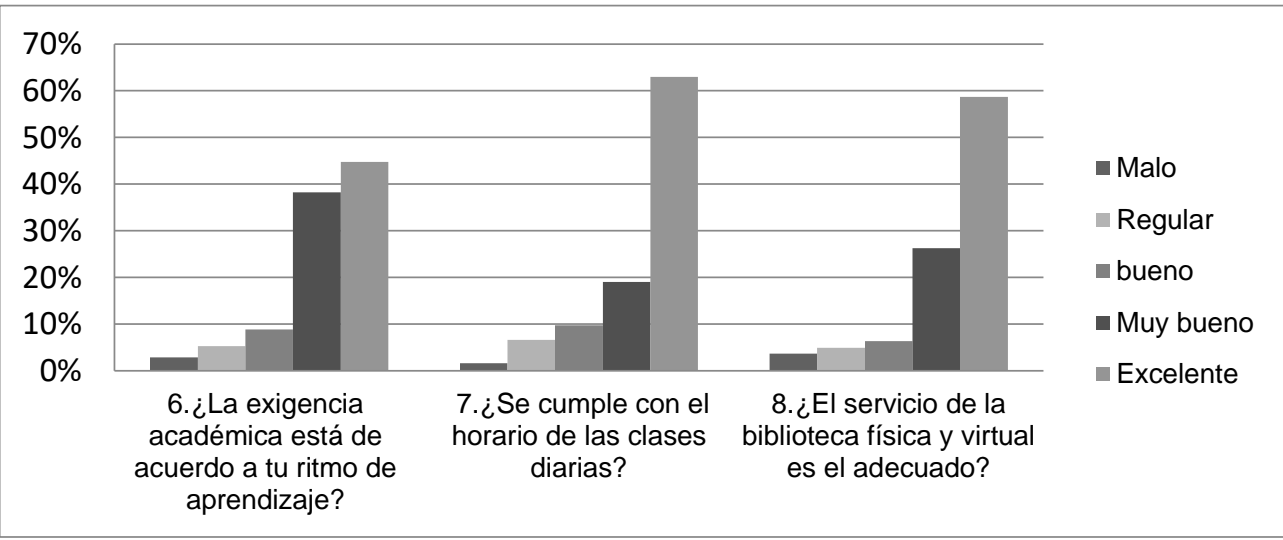

Gráfico No. 2: Condiciones institucionales (parte I)

Fuente: Elaboración propia.

Ahora bien, en el gráfico No. 2, se muestran los resultados de la dimensión condiciones institucionales, donde se puede apreciar como solo el $45 \%$ de los studiantes califican de excelente el nivel de exigencia acedémica exigido por la institución durante su proceso de aprendizaje; sin embargo, el $63 \%$ de los encuentados manifestaron que se cumple con el horario de las clases diarias; y en relación a si el servicio de la biblioteca física y virtual es el adecuado, $59 \%$ de los encuestados indicaron que es excelente. 
Gráfico No. 3: Condiciones institucionales (parte II)

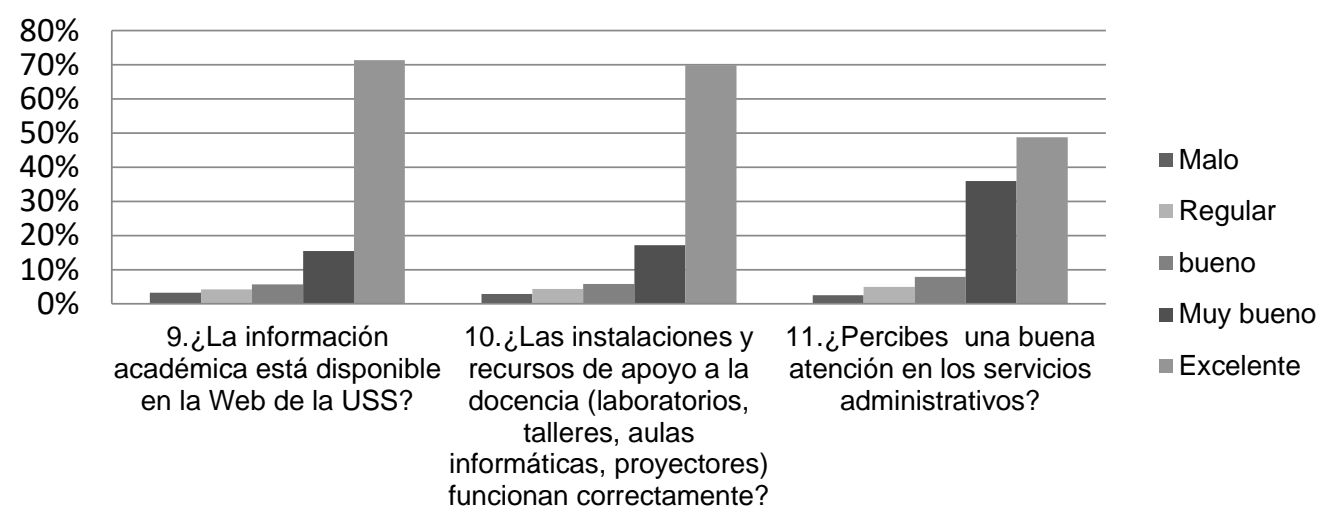

Fuente: Elaboración propia.

Seguidamente como se puede apreciar en el gráfico No.3, coincidiendo con los resultados anteriores, el $71 \%$ de los entrevistados señalaron como excelente la información académica disponible en la Web de la universidad. Respecto al funcionamiento adecuado de las instalaciones y recursos de apoyo a la docencia (laboratorios, talleres, aulas informáticas, proyectores), $70 \%$ de los estudiantes lo calificaron de excelente, no obstante solo un $49 \%$ del total de los encuestados perciben una buena atención en los servicios administrativos.

Dichos resultados presentados anteriormente en los gráficos 2 y 3 sobre las condiciones institucionales evidencian una alta calidad en relación a los recursos utilizados para el proceso formativo dentro de la Universidad, coincidiendo así con lo señalado por Teo (2019), sobre el llamado a los docentes de todo el mundo para preparar a los estudiantes para el siglo XXI y ayudarlos a navegar por un mundo cada vez más globalizado y un paisaje interconectado, junto con lo planteado por la UNESCO (1998), la gestión educativa estratégica debe estar basada en la integración de la gestión del conocimiento, la ciencia, la tecnología y la innovación en la educación.

Gráfico No. 4: Condiciones de calidad universitaria (parte I)

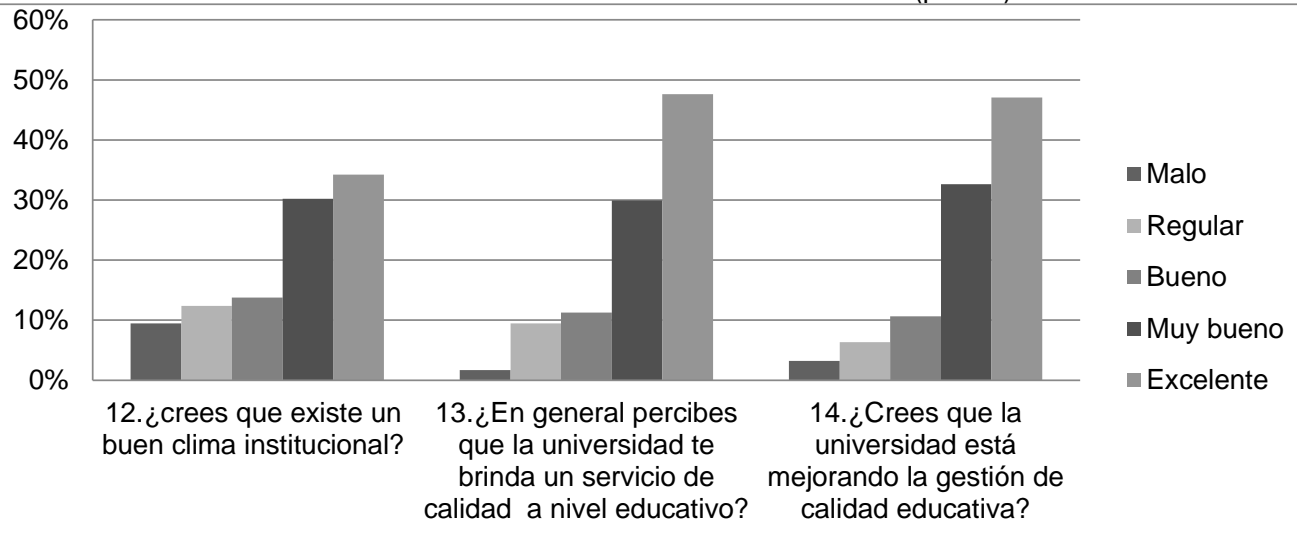


Fuente: Elaboración propia.

En el gráfico No. 4, se muestran los resultados de la dimensión condiciones de calidad universitaria, donde, respecto a la formación de calidad que brinda la Universidad Señor de Sipán, solo el $34 \%$ de los estudiantes consideran que existe un buen clima institucional, sin embargo, el $48 \%$ de los estudiantes manifestaron que perciben un servicio de calidad a nivel educativo, mientras que el $47 \%$ de los entrevistados indicaron que la universidad está mejorando la gestión de calidad educativa.

Gráfico No. 5: Condiciones de calidad universitaria (parte II)

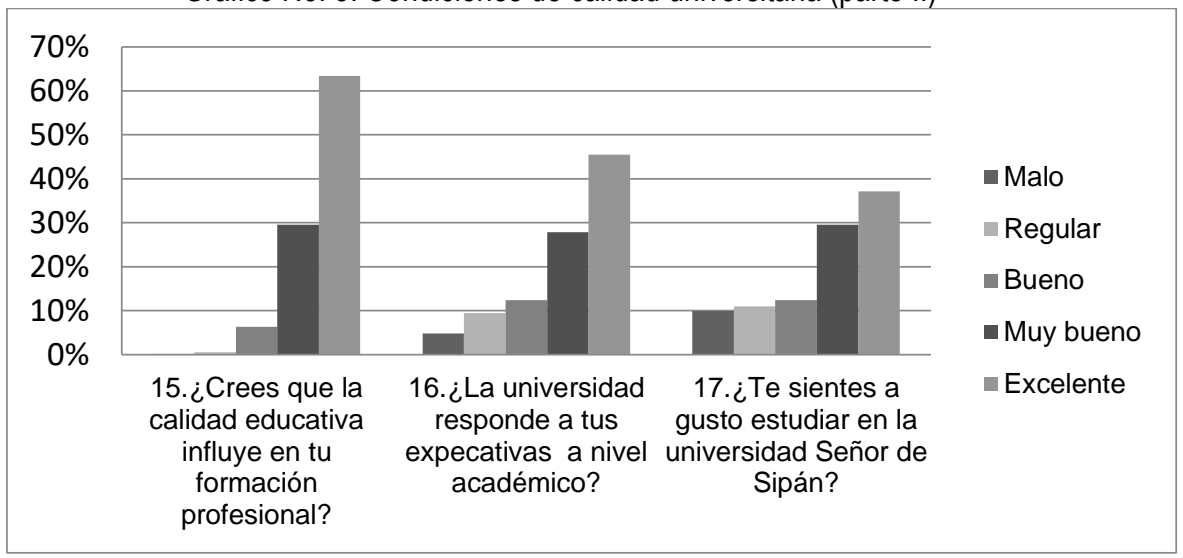

Fuente: Elaboración propia.

Por su parte, se observa en el gráfico No. 5 , como el $63 \%$ de los encuestados cree que la calidad educativa influye en su formación profesional, pero solo el $45 \%$ señaló que la universidad responde a sus expecativas a nivel académico y solo un $37 \%$ se sienten a gusto de estudiar en la universidad Señor de Sipán.

Los anteriores resultados indican que estudiar en la universidad Señor de Sipán es una alternativa para quienes buscan una formación de calidad, pues sus estudiantes catalogan de excelente las condiciones de calidad universitaria que se les brinda en la misma, siendo esto importante para el proceso de su desarrollo académico tal como lo refieren Belasha, et al. (2015), donde el componente esencial de una evaluación y garantía de calidad de un programa de educación es el monitoreo de la satisfacción de los interesados con los resultados educativos. 
Gráfico No. 6. Condiciones de asistencia a clases (parte I)

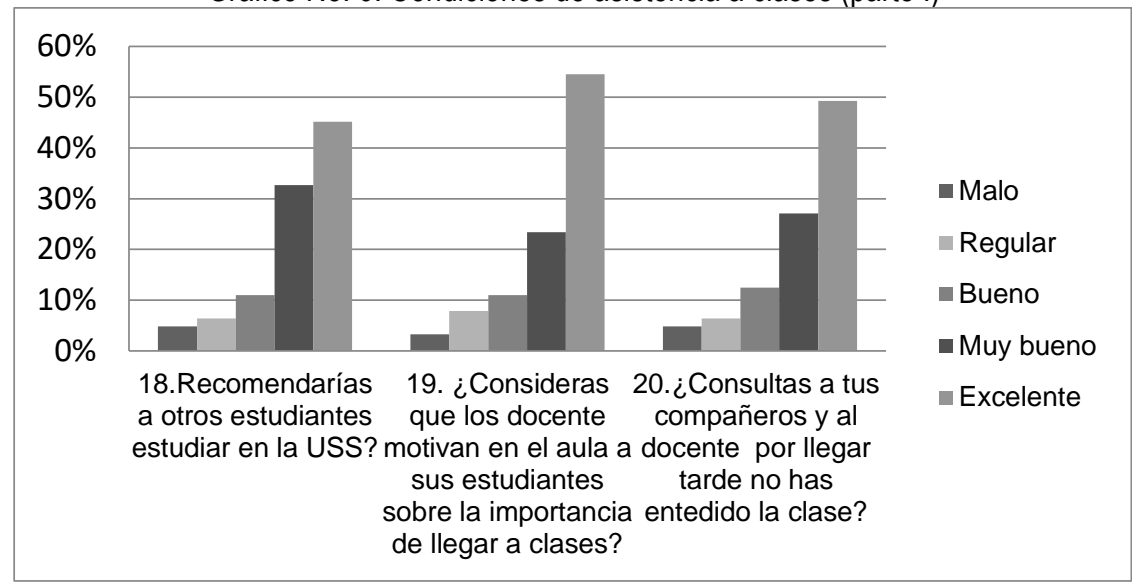

Fuente: Elaboración propia.

Ahora bien, en el gráfico No. 6 , se presentan los resultados sobre las condiciones de asistencia a clases, donde solo el $45 \%$ de los estudiantes recomendarían a otros estudiar en la USS, sin embargo, el $54 \%$ consideran que los docentes motivan, mientras que un $49 \%$ consultan a sus compañeros y al docente por llegar tarde y no haber entedido la clase. Seguidamente, como se muestra en el gráfico No. 7, el $52 \%$ de los estudiantes manifestaron llegar a clases a la hora indicada y sentirse motivados, al igual que un $45 \%$ que indicaron no faltar a clases porque lo consideran importante y si alguno faltara inmediatamente buscan ponerse al día, tal como lo evidencian el $50 \%$ de los entrevistados.

Gráfico No. 7. Condiciones de asistencia a clases (parte II)

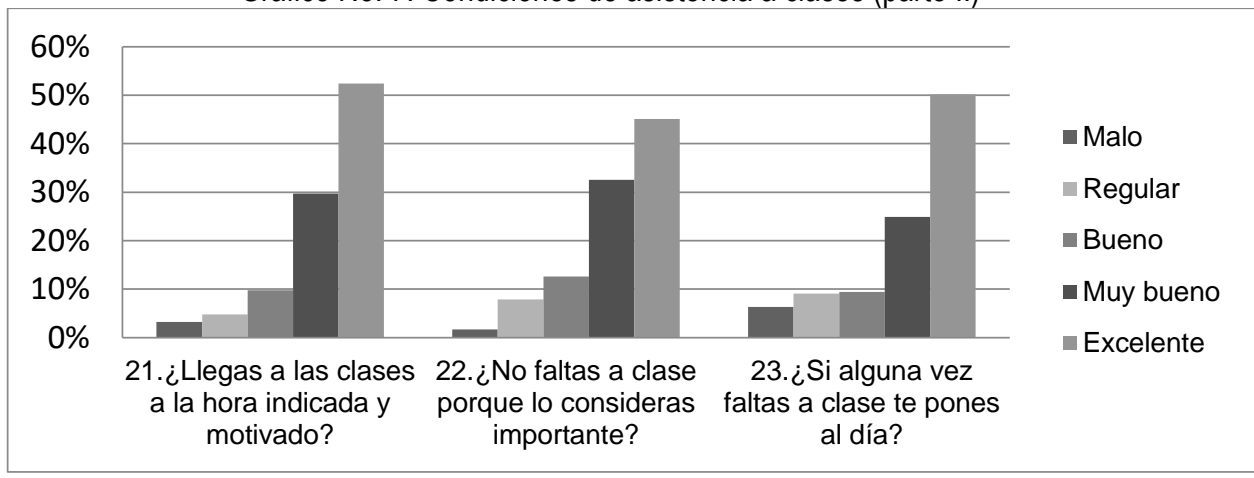

Fuente: Elaboración propia.

Los anteriores resultados demuestran que a pesar de que hasta ahora la universidad se esmera en brindar alta calidad en su formación, son los mismos estudiantes, los que llegan 
afectar la completa excelencia de la misma, pues las situaciones personales de cada estudiante, determinan su comportamiento y responsabilidad académica; limitando así, una educación óptima, confirmando los planteamientos de Hernández, et al (2017), quienes argumentan que una de las limitaciones hacia la educación de calidad son los desafíos socioeconómicos, particularmente sobre la desigualdad y la pobreza que caracteriza las familias del país, obstaculizando el crecimiento y mejores niveles de vida, incluido el acceso a la educación en sus distintos niveles, además, lo planteado por García (2012), citado por Navea y Suarez (2017), las instituciones educativas son llamadas a formar estudiantes universitarios para que se convierten en profesionales con óptima capacidad de aprender a aprender durante toda la vida en un entorno globalizado.

\section{Gráfico No. 8. Condiciones de interración estudiante-docente (parte I)}

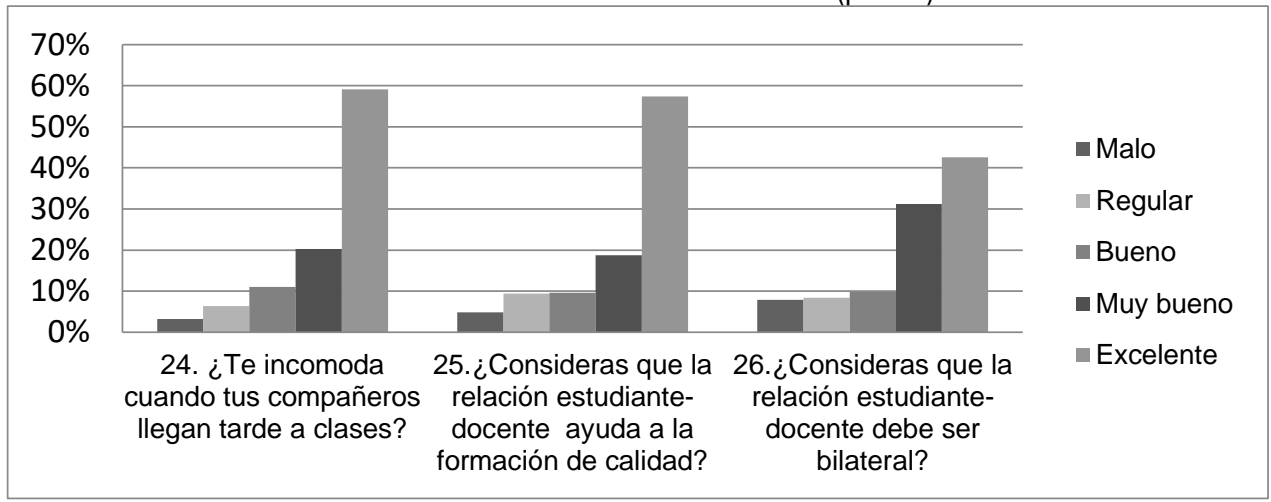

Fuente: Elaboración propia.

Por otra parte, con relación a las condiciones de interrelación estudiante-docente, se muestra en el gráfico No. 8 , que el $59 \%$ de los encuentados manifestaron su incomidad cuando los demás compañeros llegan tarde, mientras que el $57 \%$ de los estudiantes afirmaron que la relación estudiante-docente ayuda a la formación de calidad, sin embargo, el $43 \%$ considera que la relación estudiante-docente debe ser bilateral. Asimismo, en el gráfico No. 9, el 54\% de los estudiantes consideran que la relación estudiante-docente debe generar un clima de confianza, el $43 \%$ de los entrevistados afirma que la relación estudiante-docente ayuda a valorar lo que la universidad le brinda y el $40 \%$ considera que la relación estudiante-docente ayuda a la universidad a mejorar la calidad de servicios. 
Gráfico No. 9. Condiciones de interración estudiante-docente (parte II)

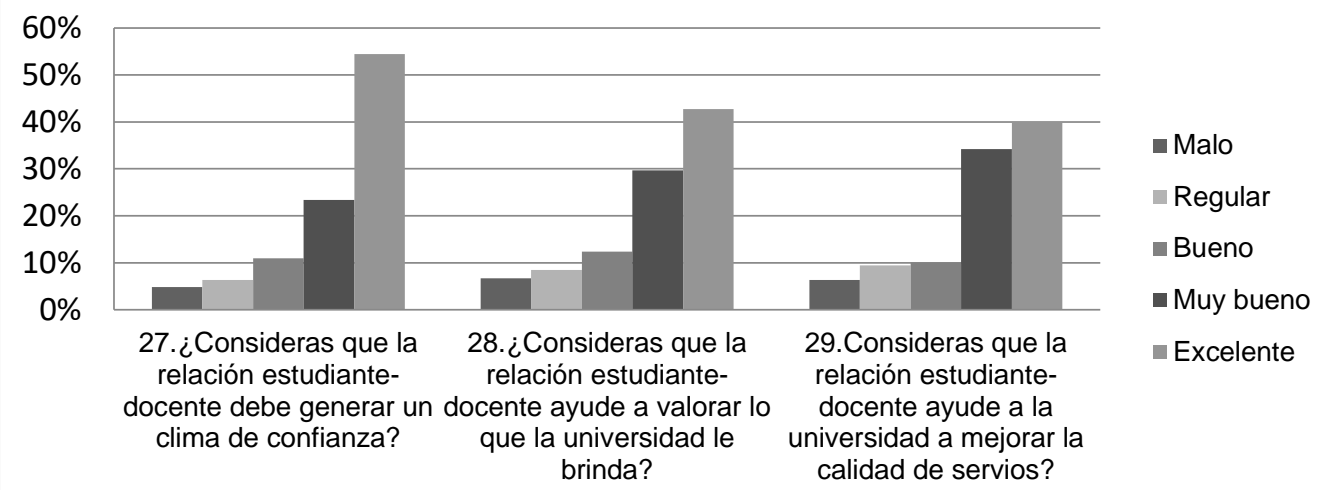

Fuente: Elaboración propia.

Los resultados mostrados anteriormente indican que los docentes están altamente capacitados en materia de metodología, didáctica y para desarrollar en los estudiantes las competencias necesarias dentro del campo laboral al cual aspira formar parte, afirmando lo referido por Gafas, et al (2018), donde, la preparación permanente e integral del docente universitario constituye un eje fundamental para la formación del estudiante y la transformación de la sociedad. Sustentado en adquisición de habilidades del lenguaje, comunicación, el pensamiento crítico, ético y del conocimiento de un idioma extranjero y de las TIC, lo que les permitirá integrar las funciones sustantivas de la universidad, guiadas por la investigación.

Finalmente, en el gráfico №. 10, se pueden apreciar los resultados obtenidos respecto a las condiciones de aptitudes que caracterizan a los estudienates de la universidad Señor de Sipan, donde, $49 \%$ de ellos considera que la relación estudiante-docente ayuda al docente a dar mejor trato a sus estudiantes, a su vez, el $52 \%$ de los encuestados indican que tienen vocación para la carrera profesional que han elegido, pero solo el $42 \%$ de los estudiantes manifestaron que tienen capacidades para la vida universitaria y el $45 \%$ creen que tienen capacidades para trabajar en equipo. 
Gráfico No. 10: Condiciones de aptitudes (parte I)

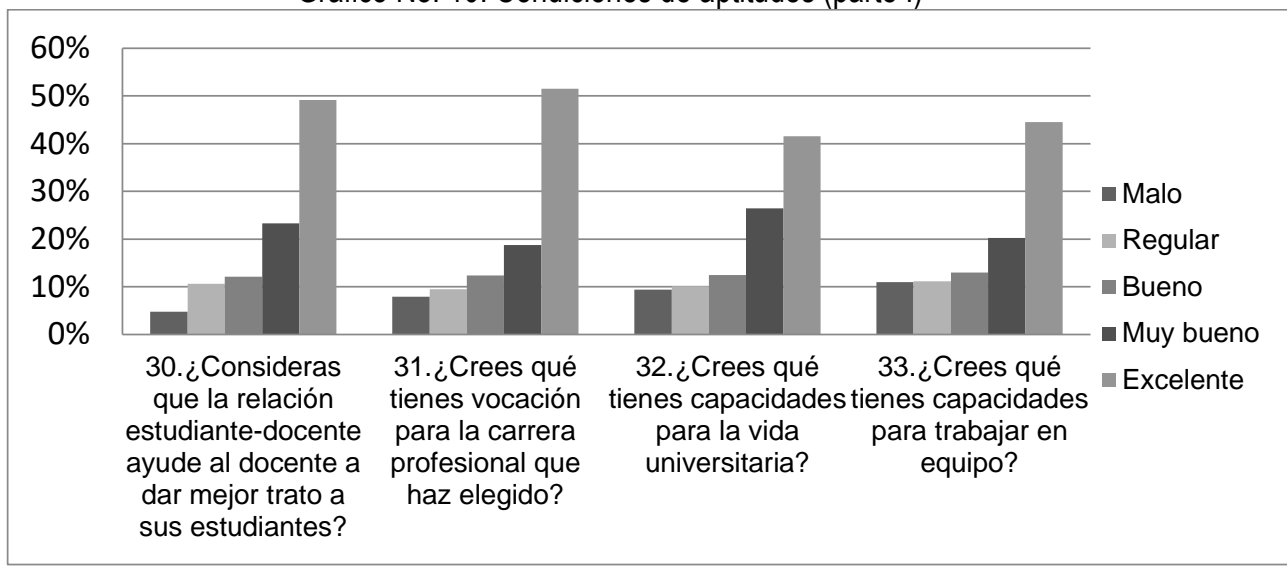

Fuente: Elaboración propia.

En este mismo orden de ideas, se observa en el grafico No. 11 , como $42 \%$ de los estudiantes cree que es importante ser responsable, hablando así muy bien de los valores personales de dichos encuestados, aunado al 39\% de los mismos que afirmó cumplir con los trabajos asignados por sus docentes, reafirmando la aptitud anterior sobre la responsabilidad que demuestran en sus obligaciones, junto al $47 \%$ de los estudiantes que afirmaron estudiar por iniciativa propia, demostrando las ganas de superación personal que poseen los estudiantes universitarios.

Gráfico No. 11: Condiciones de aptitudes (parte II)

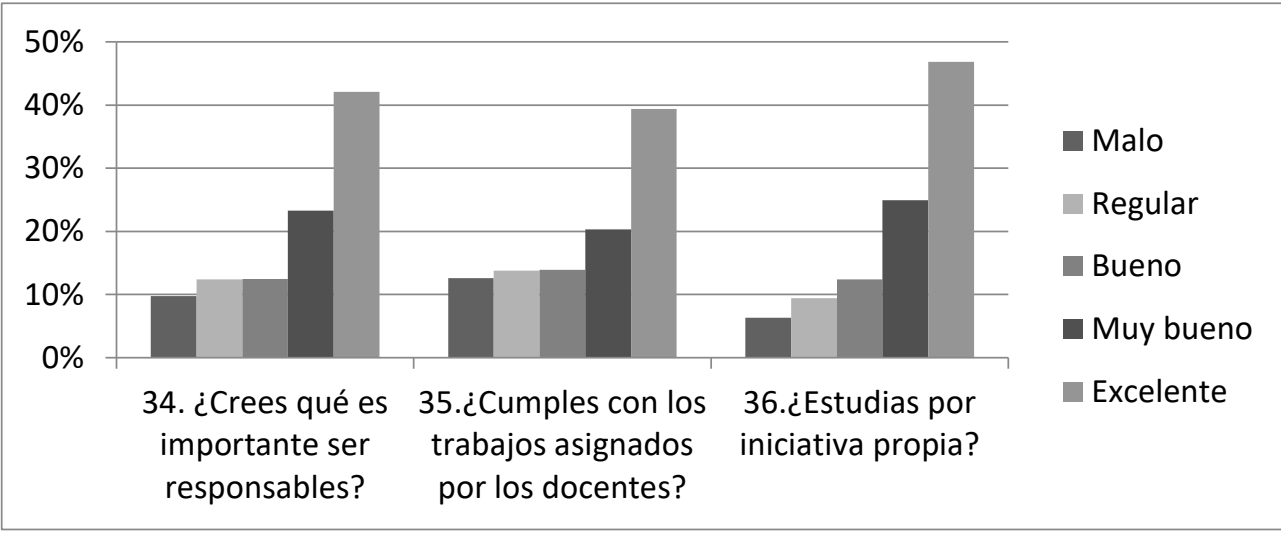

Fuente: Elaboración propia.

Dichos resultados antes expuestos confirman en cuanto a las aptitudes, un gran nivel de satisfacción personal y motivación en cuanto a la educación que reciben de las universidades 
en Perú, demostrando los fundamentos teóricos de Buchbinder (2018), pues la aspiración de todos los procesos de cambios, reformas universitarias es construir un compromiso más intenso con la vida cultural y científica de las sociedades. Por lo cual, Motefakker (2016), recomienda a las autoridades universitarias tomar medidas pertinentes para aumentar la satisfacción de los estudiantes, mejorando el nivel de servicios educativos de calidad, aunado a Shaikh, et al, (2019), quienes afirman que los docentes necesitan ampliar sus estrategias de instrucción, a fin de construir un ambiente de aprendizaje de calidad.

Todos los resultados analizados en las difentes tablas presentadas anteriormente, concuerdan en señalar un alto nivel de calidad en la formación universitaria de los estudiantes de la universidad Señor de Sipan de Perú, tomada como referencia para medir el nivel de satisfacción del estudiante sobre la calidad de formación que percibe en las instituciones de educación superior.

Es decir, con estos resultados se corrobora la hipótesis planteada en esta investigación sobre si se evaluara el nivel de satisfacción en el estudiante; entonces, se mejoraría la calidad de formación profesional en la universidad formando profesionales más competetivos, evidenciando que efectivamente si es importante evaluar la percepción del estudiante para lograr las mejoras esperadas en la gestión de calidad de formación académica.

\section{Conclusiones}

En la actualidad en el Perú existe una carencia de investigaciones que midan el nivel de satisfacción de los estudiantes en relación a la calidad educativa a nivel universitario, minimizando así la importancia de la percepción del estudiantado sobre la calidad de formación que percibe en la universidad donde desarrolla su proceso de aprendizaje. El presente artículo tuvo como objetivo analizar cómo influye el nivel de satisfacción del estudiante en relación con la formación de calidad profesional que recibe a nivel universitario

Donde, luego de la investigación realizada se pudo evidenciar que los estudiantes en su gran mayoría perciben un alto nivel de satisfacción en relación con la formación de calidad universitaria recibida en su institución, sin embargo, existe otro porcentaje minoritario de estudiantes que señala algunas fallas relacionadas con las condiciones de calidad, didácticas de interrelación alumno-docente, de aptitudes propias del estudiantado, entre otros aspectos que deben ser tomados en cuenta para seguir optimizando la calidad del servicio ofrecido en cada institución de educación superior.

Por lo cual, los resultados obtenidos de la presente investigación debe ser base para la formulación y evaluación de las políticas de cada universidad, asimismo deben ser consideradas por el ministerio de educación para seguir trabajando en mejorar no solo la calidad educativa sino la percepción que el estudiante tiene frente a ella. La universidad debe estar comprometida con la sociedad, dar cabida a la crítica, reflexión y generar procesos de transformación acordes con los requerimientos de seres humanos, del avance de su historia, que precisan también innovar e incorporar los avances de la ciencia y tecnología en su proceso de enseñanza académica y universitaria, con programas debidamente actualizados conforme cambian las exigencias empresariales y de las organizaciones donde cada egresado ejercerá su profesión.

A su vez, es importante resaltar que dentro de éste ámbito universitario, existe preocupación por el personal docente y su interacción directa con la producción y la difusión 
del conocimiento, por lo cual, se hace necesario principalmente formentar un alto desempeño del docente como investigador y seguidamente, fomentar la creación de espacios para la reflexión que conduzcan a su formación pedagógica e investigativa, en procura de una educación con calidad, comprometida con los estándares exigidos por el sistema educativo y cumpliendo las expectativas generadas por la sociedad.

\section{Referencias Bibliográficas}

Adie, Jean-Paul; Acuto, Michele; Ho, K.C; Cairns, Stephen y PinkTan, Hwee. (2019). Perspectives on the 21st Century Urban University from Singapore - A viewpoint fórum. Elsevier Cities, 88, Washington. (Pp. 252-260). https://doi.org/10.1016/j.cities.2018.11.006

Alvarado Peña, Lisandro y Moreno Freites, Zahira. (2017). Gobernanza y gestión de calidad en los centros de investigación universitarios. Revista Opción, 33 (84), Venezuela. (Pp. 471-503).

Alvarado Peña, Lisandro e Izaguirre Díaz, Fridzia. (2015). Calidad del servicio en universidades públicas mexicanas: perspectiva de análisis desde las dimensiones. Revista pensamiento y gestión, (38), Colombia. http://dx.doi.org/10.14482/pege.38.7702.

Argimón Pallás, Josep y Jiménez Villa, Josep. (2013). Métodos de investigación clínica y epidemiología. (Cuarta edición). Editorial Elsevier. Barcelona, España.

Arias Odón, Fidias (2016). El proyecto de investigación. Inducción a la metodología científica. (Séptima edición). Editorial episteme. Caracas, Venezuela.

Barbón Pérez, Olga. (2018). The role of strategic educational management in knowledge management, science, technology, and innovation in higher education. Elsevier Educación Médica, 19, (1), España. (Pp. 51-55. https://doi.org/10.1016/i.edumed.2016.12.001.

Belasha, Olga; Popov, Mikhail; Ryzhov, Nicolai; Yan, Ryaskov; Shaposhnikov, Sergey; Shestopalov, Mikhail. (2015). Research on University Education Quality Assurance: Methodology and Results of Stakeholders' Satisfaction Monitoring. Elsevier Procedia - Social and Behavioral Sciences, 214, United Kingdom (Pp 344-358). Extraido de: https://cyberleninka.org/article/n/875889/viewer.

Benavides Abanto, Martín. (2018). Van 36 universidades licenciadas. Extraido de: https://educacionalfuturo.com/noticias/van-36-universidades-licenciadas/.

Betlloch, Mas; Sapena, Ramón; García, Abellán y Ramirez, Pascual. (2019). Implementation and Operation of an Integrated Quality Management System in Accordance With ISO 9001:2015 in a Dermatology Department. Actas Dermo-Sifiliográficas, 110, España. (Pp. 92-101). https://doi.org/10.1016/j.adengl.2019.01.003

Brewa, Angela y Saunders, Constanze. (2020). Making sense of research-based learning in teacher education. Elsevier Teaching and Teacher Education, 87, United Kingdom. (Art. 102935). DOl: https://doi.org/10.1016/j.tate.2019.102935.

Bruns, Barbara; Harbaug Macdonald, Isabel y Schneider, Ben. (2019). The politics of quality reforms and the challenges for SDGs in education. Elsevier World Development, 118, París. (Pp. 27-38). https://doi.org/10.1016/i.worlddev.2019.02.008. 
Brunner, José y Ganga, Francisco. (2018). Reforma a la educación superior: un análisis crítico del enfoque chileno. Revista Utopía y Praxis Latinoamericana. 23 (83), Venezuela. (Pp. 138-146). http://doi.org/10.5281/zenodo.1438580.

Buchbinder, Pablo. (2018). Thinking the university reform one hundred years later. Revista Iberoamericana de Educación Superior, 9, (25), México. Extraido de: http://www.scielo.org.mx/scielo.php?script=sci_abstract\&pid=S2007-

28722018000200086\&lng=es\&nrm=iso\&tlng=en.

Bulut, Nergz; Serin, Oguz y Özbaş, Filiz. (2010). Predicting university students' life satisfaction by their anxiety and depression level. Elsevier Procedia - Social and Behavioral Sciences, $\quad 9, \quad$ Netherlands. https://doi.org/10.1016/j.sbspro.2010.12.200.

Foon, Khe; Hu, Xiang; Quia, Chen y Tang, Ying. (2020). What predicts student satisfaction with MOOCs: A gradient boosting trees supervised machine learning and sentiment analysis. Elsevier Computers Education, 145, United Kingdom. https://doi.org/10.1016/j.compedu.2019.103724.

Gaete Quezada, Ricardo. (2015). The glass ceiling in public universities in Chile. An exploratory analysis. Revista Iberoamericana de Educación Superior, 6, (17), México. (Pp. 3-20). https://doi.org/10.1016/i.rides.2015.06.001.

Gafas Gonzalez, Carlos; Herrera Molina, Angelica; Brossard Peña, Edgar; Roque Herrera, Yosbanys y Ferrera Larramendi, Ramón. (2018). Higher education in the health sciences. The Ecuador context. Elsevier Educación Médica, 19, (1), España. (Pp. 34-38). Extraido de: https://doi.org/10.1016/i.edumed.2016.08.006.

García Jimenez, Eduardo. (2016). A definition of excellence in higher education. Elsevier Educación Médica, 17, (3), España. (Pp. 83-87). https://doi.org/10.1016/j.edumed.2016.06.003

Hernández Brigans, Hector; Martusce Quintana,Jaime; Moctezuma Navarro, David; Muñoz García, Humberto y Narro Robles, José. (2015). Los desafíos de las universidades de América Latina y el Caribe ¿Qué somos y a dónde vamos?. Perfiles Educativos, 37, (147), México. (Pp. 202-218). https://doi.org/10.1016/j.pe.2015.11.001.

Mejía, Julio. (2018). The process of higher education in Perú. The decoloniality of university knowledge. Cinta de moebio, (61), Chile. (Pp:56-71). http://dx.doi.org/10.4067/S0717-554X2018000100056.

Motefakker, Niloofar. (2016). The Study of the Level of Satisfaction of the Students of the Faculty of Social Sciences with Welfare Services of Imam Khomeini International University of Qazvin. Elsevier Procedia Economics and Finance. 36, Amsterdam. (Pp. 399-407). DOI: https://doi.org/10.1016/S2212-5671(16)30052-1.

Navea Martín, Ana; Suarez Riveiro, José. (2017). Estudio sobre la utilización de estrategias de automotivación en estudiantes universitarios. Psicología Educativa, 23, (2), Madrid. (Pp. 115-121). https://doi.org/10.1016/i.pse.2016.08.001

Osborne, Jonathan. (2013). The 21st century challenge for science education: Assessing scientific reasoning. Elsevier Thinking Skills and Creativity, (10), Netherlands. (Pp. 265-279). https://doi.org/10.1016/j.tsc.2013.07.006.

Real Academia Española (RAE, 2010). Ortografía de la lengua española. Editorial: Espasa Libros. España. 
Rivero Bottero, Raquel. (2013). Educación y Pedagogía en el marco del neoliberalismo y la globalización. Revista Perfiles Educativos. 35 (142), México. (Pp. 149-166). Extraido de: https://www.sciencedirect.com/science/article/pii/S018526981371854

Sánchez Quintero, Jairo. (2014). A proposal of quality indicators to self-assessment and accreditation of undergraduate programs in management. Estudios Gerenciales, 30, (133), Colombia. (Pp. 419-429). https://doi.org/10.1016/j.estger.2014.06.002.

Şen, Asim; Erkan Kabak, Kamil; Tüysüz, Fatih; Kuzaliç, Dilek. (2012). Democratization of University Management for Quality Higher Education. Elsevier Procedia - Social and Behavioral Sciences, 58, Netherlands. (Pp. 1491-1504). Extraido de: https://cyberleninka.org/article/n/529283/viewer.

Shaikh, Adnal; Aldarmahi, Ahmed; Al-Sanie, Ebtehal; Subahi, Ahmad; Ahmed, Mohamed; Hydrie, Mohd; Al-Jifree, Hatim. (2019). Learning styles and satisfaction with educational activities of Saudi Health Science University Students. Journal of Taibah University Medical Sciences. 14,(5), Arabia Saudita. (Pp: 418-424). https://doi.org/10.1016/i.jtumed.2019.07.002

Sutherland, Dylan; Warwick, Philip y Anderson, John. (2019). What factors influence student satisfaction with module quality? A comparative analysis in a UK business school context. Elsevier The International Journal of Management Education, 17, (3), Netherlands. (Pp: 1-30). DOI: https://doi.org/10.1016/i.jime.2019.100312.

Teo, Peter. (2019). Teaching for the 21st century: A case for dialogic pedagogy. Elsevier Learning, Culture and Social Interaction, 21, Netherlands. (Pp. 170-178). https://doi.org/10.1016/j.lcsi.2019.03.009

Trujillo Segoviano, Jorge. (2014). El enfoque en competencias y la mejora de la educación. Revista Ra Ximhai, 10 (5), México. (Pp. 307-322). Extraido de: https://www.redalyc.org/pdf/461/46132134026.pdf.

UNESCO. (1998). La educación superior y Sociedad.Conferencia Mundial sobre la Educación Superior. La educación superior en el siglo XXI: Visión y acción. Extraido de: https://www.iesalc.unesco.org/ess/index.php/ess3/article/view/171.

Vilcea, Mihai. (2014). Quality Culture in Universities and Influences on Formal and Non-formal Education. El sevier Procedia - Social and Behavioral Sciences, 63 (19), United Kingdom. (Pp 148-152). https://doi.org/10.1016/..sbspro.2014.12.300 